\title{
When China Encounters Smart TV: Exploring Factors Influencing the User Adoption in China
}

\author{
Yuming Tao, Jing Chang, and Pei-Luen Patrick Rau \\ Department of Industrial Engineering, Tsinghua University, Beijing 100084, P.R. China \\ \{taoym08, changj12\}@mails.tsinghua.edu.cn, \\ rpl@tsinghua.edu.cn
}

\begin{abstract}
Smart TVs, providing users with the access to abundant number of Internet video contents as well as other 'smart' functionalities, are gradually becoming more and more popular within Chinese households. Chinese internet companies and TV manufactures has been actively developing two forms of smart TV, namely embedded smart TVs and smart set-top boxes. The objective of the study is to explore the factors that affect user adoption of both the embedded smart TV and the set-top box and comprehensively analyze the difference in user adoption of the two smart TV forms. We carried out an online survey and collected 245 valid responses. With the help of exploratory factor analysis and hierarchical multiple regression analysis, we built a smart TV user adoption model based on TAM with 6 antecedent factors. The model helps to explain the difference between user adoption of embedded smart TV and smart set-top box and also figured out that perceived usefulness on video functionality is the priority concerns for developing both embedded smart TV and smart settop box.
\end{abstract}

Keywords: Smart TV, Set-top box, Technology Acceptance Model.

\section{Introduction}

As an information and entertainment medium that has traditionally occupied the largest share of leisure time [1], TV has the greatest potential after mobile phone to bring about a revolution to the internet industry. Smart TVs enable users to have infinite access to Internet videos and applications in living room, making it easier for people to share Internet contents together with their families and friends. Smart TV differentiates itself from a traditional TV by integrating an OS platform, which grants a smart TV with all its smart functionalities.

A smart TV can come into two forms: an embedded smart TV set or a traditional TV branched with a smart set-top box. Even though the two forms of smart TVs are similar in terms of their functionalities as well as their user experiences, installation of the two forms are quite different to Chinese users. Installing a smart TV means the replacement of previous TV set, and the price to be paid can go from several thousands to tens of thousands RMB. In comparison, installing a set-top box is a much cheaper alternative, and the prices go between 200 - 500 RMB in general cases. 
According to a research by Liu et al., the cost is amongst the most important factors that influence the user acceptance of mobile entertainment of Chinese rural users. [2] In their study, Liu further attributed this phenomenon to the social-economic structure in China. So far, no studies of this kind were carried out in the case of smart TV. We expected the intention to adopt the two forms of smart TV will be also very different to Chinese users particularly in the less developed area, and we meant to explore it in this study.

Smart TVs has witnessed considerable growth in China since 2012. The Chinese big internet companies, including Baidu, Alibaba, Huawei and Xiaomi, has all launched their own embedded smart TV products or smart set-top boxes in recent two years. The TV manufactures were also actively taking part in this newly emerged market. Some expected that the era of Smart TVs would come at a much faster rate following the success of the smartphone. There are also prediction that smart TVs would become a hub at home by connecting computers and telephones in homes and automating electricity, home security and other entertainment systems [3].

While smart TV nowadays provide various kinds of so called 'smart' functionalities to its users, for example, online video streaming, TV gaming, online shopping \& hotel booking, website browsing, etc., no one is sure of what kind of functionalities lead Chinese consumers to adopt this device. Yet, besides these seemingly 'powerful' functionalities provided by smart TVs, Chinese consumers seem to adopt smart TVs for other reasons, for example, the feeling of self-esteem as the early adopters of the device 'in fashion', or be persuaded by friends and relatives. It's still unknown which are the main factors that influence Chinese users' adoption of the forms of smart TV respectively, and how to accordingly optimize the current smart TV products to make it more concurrent amongst its peers.

The objective of our research is to explore the factors that influence the user adoption of smart TV in China, including the personal factors (user demographic characteristics, past experiences with smart devices, user innovativeness, etc.), social factors (social influence, etc) and product property factors (perceived product quality, relative advantage, etc.). We aimed to build up a comprehensive user adoption model for both embedded smart TV and smart set-top box. The results will help to explain the following questions which have bothered the industry for many years.

1. What kind of people is most likely to adopt embedded smart TV and smart set-top box?

2. What are the most important property factors that influence the adoption of embedded smart TV and smart set-top box?

3. How the intention of adoption is affected by social factors like social influence?

The study provides both theoretical and industrial contributions. Theoretically, it is the first comprehensive smart TV user adoption model that distinguishes the differences between embedded smart TV and smart set-top box, particularly under the social-economic situation in China. For the industry, it helps to figure out the potential smart TV adopters. It also helps to gain insights on how to optimize the current TV products and how to exert external influences over the potential user to accelerate the process of adoption. 


\section{Theoretical Foundation and Research Framework}

\subsection{Technology Adoption Theories}

Technology acceptance or technology adoption theories has been studied for tens of years, and there're two theoretical model has been most widely used: the technology acceptance model (TAM) [4] and the innovation diffusion theory (IDT) [5].

TAM was initially developed to predict user acceptance of computers. According to Davis [4], user acceptance is fundamentally determined by two specific variables, perceived usefulness and perceived ease of use. Perceived usefulness is defined as "the degree to which a person believes that using a particular system would enhance his or her job performance". Perceived ease of use, in contrast, refers to "the degree to which a person believes that using a particular system would be free of effort." This model is a convergence of Self-efficacy theory [6], Cost-benefit paradigm [7], and Tornatzky and Klein's [8] findings about the adoption of innovations.

Another important model for an innovation's user acceptance is the innovation diffusion theory (IDT) [5]. According to Roger, diffusion is the process by which an innovation that is communicated through certain channels over time among the members of a social system. A new innovation is always adopted firstly by some early adopters, and then spreads through some social channels. According to the innovation diffusion theory, there are five other elements that affect the adoption rate of an innovation, namely the relative advantage, compatibility, complexity, trialability and observability.

\subsection{Research Framework}

In our study, we tried to extend the TAM model. Perceived usefulness (PU) and perceived ease of use (PEOU) were expected to be directed related to intention to use (ITOU), and we aimed to explore the antecedents that will influence PU and PEOU. Based on the previous theories and research, we concluded 5 candidate factors that will affect PU and PEOU, namely compatibility (COM), relative advantage (RA), innovativeness (INV), product quality (PQ) [9]. Although, after interviewing several experts in the smart TV industry, we come up with two other factors: past experiences with other smart devices and willingness to share TV contents with families.

Past experience with other smart devices (PE) was expected to have two-fold effect on the intention to adopt smart TV. On the one hand, subjects which have satisfying experiences in using smart phones or smart tablets were expected to have better understanding of the smart functionalities that smart TV provides, thus have a higher PU and PEOU. However, on the other hand, if the subject has satisfying past experience with smart phones or smart tablets, he/she might find smart TV redundant in terms of functionality, because most the 'smart' functionalities that smart TV provide, has already been realized on smart phones and smart tablets. Thus, PE could also have negative impact on PU and PEOU.

Willingness to share TV contents with families ( $\mathrm{SH}$ ) was regarded as another factor that will influence the intention to adopt smart TV. Different from other personal devices like PC and smart phone, TV is better suited for multi-user situation. A higher willingness to share TV contents with families may result in higher PU. 


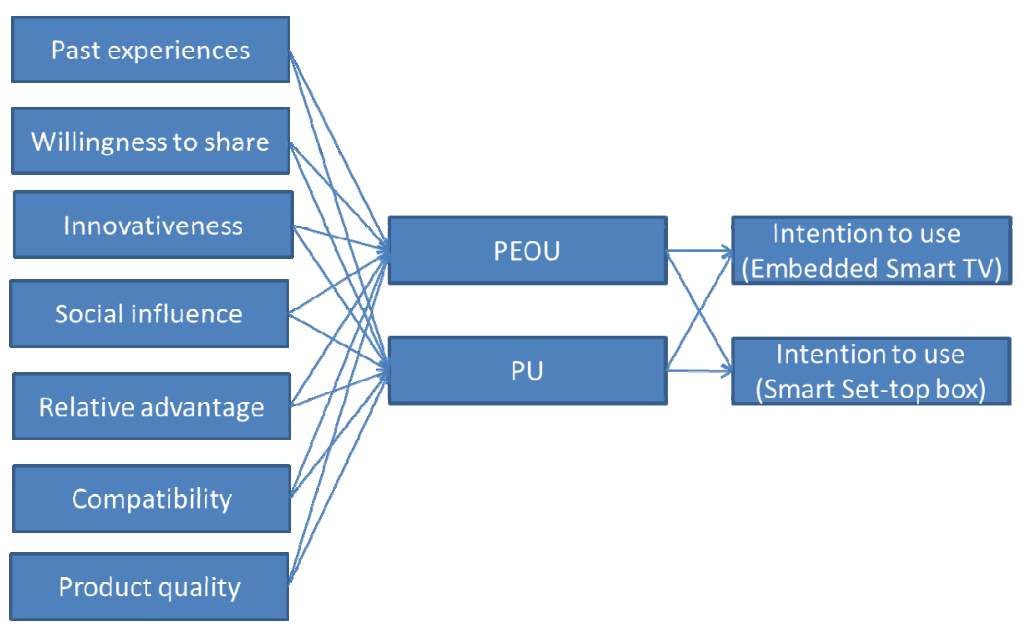

Fig. 1. Research framework for smart TV user adoption

\section{Survey Study}

Based on the research framework proposed previously, we developed the questionnaire for our survey. The questionnaire consisted 3 parts. The first part is constituted of the questions on personal profile, including gender, age, education level, household size and if the subject is currently using an embedded smart TV or smart set-top box. The second part is constituted of the questions on subject's usage of PC and smart phone. The third part is constituted of a total of 48 7-likert scale items for the 9 antecedent factors of intention to use in the framework. The subjects' intention to use embedded smart and smart set-top box was asked at the end of the survey.

The items for the 6 factors namely innovativeness [10, 11], social influence [12], relative advantage [5, 13, 14], compatibility [2, 5], product quality [2, 5]. For the 2 factors, perceived usefulness (PU) and perceived ease of use (PEOU), we further divided them into several sub-dimensions in regards to different functionalities a TV provide. The different functionalities considered in our research are: online video on demand, online live streaming, TV games, TV shopping, TV online browsing. For the two factors past experiences and willingness to share, we constructed the items under the guidance of several experts of human computer interaction as there're no previous literatures discussing these factors.

An online survey was conducted on the professional questionnaire website Sojump (www.sojump.com) from December 24, 2012 to January 2, 2013. We posted the hyperlink through BBS, social network, email in order to spread the survey to across China. In order to prevent duplicate submits each unique IP address was authorized to summit the response once.

A total of 245 valid responses (45.7\% effective rate) were collected for this study. The invalid samples were filtered out based on the completion time (less than 300 seconds) and the consistency of the responses over two pairs of contrary questions. 


\section{Results Analysis}

\subsection{Sample Profile}

Table 1 shows the general profile as well as the demographic characteristics of sample respondents. Of all the 245 subjects, $56.3 \%$ were male and $43.7 \%$ were female. Most of the respondents were aged less than $40(77.2 \%)$ and a majority of them had received college education. Only $48.6 \%$ of all the respondents were currently using an embedded smart TV or smart set-top box and among them 76 were using an embedded smart TV.

Table 1. Demographic profiles of sample respondents

\begin{tabular}{llll}
\hline & Category & Frequency & Percentage \\
\hline Gender & Male & 138 & $56.3 \%$ \\
& Female & 107 & $43.7 \%$ \\
\hline Age & $\leq 29$ & 130 & $53.1 \%$ \\
& $30-39$ & 59 & $24.1 \%$ \\
& $40-49$ & 48 & $19.6 \%$ \\
\hline Educational Level & $\geq 50$ & 8 & $3.3 \%$ \\
& High School or below & 52 & $21.2 \%$ \\
& College & 131 & $53.5 \%$ \\
\hline Occupation & Master or higher & 62 & $25.3 \%$ \\
& Student & 66 & $26.9 \%$ \\
& Specialist & 44 & $18.0 \%$ \\
& Teacher & 26 & $10.6 \%$ \\
& Technician & 18 & $7.4 \%$ \\
& Executive & 16 & $6.5 \%$ \\
& Sales & 9 & $3.7 \%$ \\
& Others & 66 & $26.9 \%$ \\
\hline Household size & 1 & 24 & $9.8 \%$ \\
& 2 & 24 & $9.8 \%$ \\
& 3 & 123 & $50.2 \%$ \\
& 4 & 53 & $21.6 \%$ \\
& $\geq 5$ & 21 & $8.6 \%$ \\
\hline Current Usage of & Traditional TV user & 126 & $51.4 \%$ \\
& Embedded smart TV user & 76 & $31.0 \%$ \\
& Smart set-top box user & 43 & $17.6 \%$ \\
\hline
\end{tabular}

\subsection{Group Compare}

In order to find out what kind of people have a higher intention to use smart TV, we compared the average Intention to use (ITOU) between different groups. 
While comparing between three groups of subjects: embedded smart TV users, smart set-top box users and traditional TV users, their intentions to adopt an embedded smart TV in the future were significantly different (one way ANOVA, F=10.067, $\mathrm{p}<0.001)$. Smart TV users have the highest intention to use, while smart box users go after. As well smart box users are more likely to buy a smart TV in the future than the regular TV users. $(\mathrm{t}=2.212, \mathrm{p}=0.028<0.05$ two tailed $)$.

\subsection{Exploratory Factor Analysis}

We used a screening process to perform an exploratory factor analysis over the total of 48 likert-scale question items of the survey. Factors with loadings greater than 0.80 were retreated, with the following rules followed in the screening process.

- The cumulative sum of squared variance should be as large as possible. [15]

- Variables with loading smaller than 0.50 on all common factors should be deleted; Variables with loading larger than 0.50 on two or more factors should be deleted. [16]

- If a factor contains only one variable, the factor should be merged with other factors or be deleted. [17]

- The extracted model should be simple and easy to explain from a professional point of view. [15]

With the help of EFA and after the rotation of varimax, instead of retreating 9 constructs as expected, we had retreated 10 constructs from 27 items and the other 21 items were deleted.

The items for PU were however divided into two sub-factors after EFA: one factor is related to perceived usefulness of video functionality (PU-V), and the other for the perceived usefulness of non-video 'smart' functionalities (PU-NV). This was unexpected when constructing the survey but it showed that the subjects distinguish the video functionality from other 'smart' functionalities. By comparing the means of PU-V and PU-NV, the mean of PU-V is significantly higher than that of PU-NV (Paired t-test, $\mathrm{t}=8.979, \mathrm{p}<0.001$ ), showing that video functionalities is perceived more useful than the others.

The 10 factors had explained a total of $72.50 \%$ variances. The KMO were 0.757 and the Bartletts' Tests have significances less than 0.001, which demonstrated that the method of factor analysis was appropriate. The Cronbach's alpha value for all the construct are above 0.60 , which was considered appropriate for exploratory research. [16]

\subsection{Predicting Perceived Ease of Use}

We performed a hierarchical multiple regression analysis to test how PEOU are affected by SI, PQ, SH, PE, RA, INV and COM. Also the demographic characteristics including the current usage of smart TV as well as the age group of the user was also taken into analysis. The results are shown in the table below. 
Table 2. Hierarchical regression analysis predicting PEOU

\begin{tabular}{cccc}
\hline Predictor & $\begin{array}{c}\text { Incremental } \\
\text { (adjusted) } \mathrm{R}^{2}\end{array}$ & $\begin{array}{c}\text { Standardized } \\
\text { Coefficients }(\beta)^{1}\end{array}$ & \multicolumn{1}{c}{ Sig. ${ }^{1}$} \\
\hline PQ & $\mathbf{0 . 0 5 4}$ & $\mathbf{0 . 2 1 1}$ & $\mathbf{0 . 0 0 1} * *$ \\
SH & $\mathbf{0 . 1 4 8}$ & $\mathbf{- 0 . 4 5 0}$ & $<\mathbf{0 . 0 0 1} * * *$ \\
PE & $\mathbf{0 . 0 4 2}$ & $\mathbf{0 . 2 3 0}$ & $<\mathbf{0 . 0 0 1} * * *$ \\
INV & 0.001 & -0.071 & 0.254 \\
RA & -0.003 & 0.034 & 0.622 \\
COM & -0.003 & 0.013 & 0.840 \\
SI & -0.003 & -0.014 & 0.838 \\
\hline Age & 0.005 & -0.096 & 0.099 \\
Current Usage of & -0.002 & -0.023 & 0.694 \\
Smart TV & & & \\
\hline Total & $\mathbf{0 . 2 3 9}$ & & \\
\hline
\end{tabular}

$* \mathrm{p}<0.05 ; * * \mathrm{p}<0.01 ; * * * \mathrm{p}<0.001$.

It shows that willingness to share the TV contents with the family ( $\mathrm{SH}$ ) is a very strong predictor for perceived ease of use (contributing 14.8\% to the total variance). Product quality (PQ) and past experience with other smart devices (PE) are two other strong predictors (contributing a total of $9.6 \%$ to the variance). Demographic characteristics had no significant impact over user's perceived ease of use.

PE and PQ Have Significant Positive Impact on the PEOU. A higher PE reflects that the subject is quite familiar with the operations on smart devices, which leads to a higher PEOU. Better product quality also leads to a higher PEOU, which is coherent with our assumptions and the results of other researchers.

SH Have Significant Negative Impact on the PEOU. This is contrary to our previous assumption, and a probable reason is that a certain mediator exists between $\mathrm{SH}$ and PEOU but not yet discovered in this study. Smart TVs today may not be easy enough to use, thus those who want to share video contents with families may seek for alternatives other than smart TV and finally find that PEOU of smart TV is relatively lower compared to its alternatives.

\subsection{Predicting Perceived Usefulness}

We also performed a hierarchical multiple regression analysis on PU-V and PU-NV, the results are shown in tables below. Age and current usage of smart TV were combined into one hierarchical level. 
Table 3. Hierarchical regression analysis predicting PU-V

\begin{tabular}{cccc}
\hline Predictor & $\begin{array}{c}\text { Incremental } \\
\text { (adjusted) } \mathrm{R}^{2}\end{array}$ & $\begin{array}{c}\text { Standardized } \\
\text { Coefficients }(\beta)^{1}\end{array}$ & Sig. $^{1}$ \\
\hline PQ & 0.042 & -0.039 & 0.501 \\
SH & $\mathbf{0 . 1 4 9}$ & $\mathbf{0 . 1 7 0}$ & $\mathbf{0 . 0 1 2} *$ \\
PE & $\mathbf{0 . 0 3 3}$ & $\mathbf{0 . 1 3 3}$ & $\mathbf{0 . 0 2 4} *$ \\
INV & 0.000 & -0.011 & 0.850 \\
RA & $\mathbf{0 . 0 9 0}$ & $\mathbf{0 . 2 9 3}$ & $<\mathbf{0 . 0 0 1} * * *$ \\
COM & -0.003 & -0.052 & 0.377 \\
SI & $\mathbf{0 . 0 4 8}$ & $\mathbf{0 . 2 7 3}$ & $<\mathbf{0 . 0 0 1} * * *$ \\
PEOU & -0.002 & -0.006 & 0.921 \\
\hline Age & & 0.064 & 0.923 \\
Current Usage of & -0.002 & 0.005 & 0.234 \\
Smart TV & & & \\
\hline Total & $\mathbf{0 . 3 5 5}$ & & \\
\hline p $<0.05 ; *$ p $<0.01 ; * * * p<0.001$. &
\end{tabular}

The result in Table 3 shows that willingness to share $(\mathrm{SH})$, relative advantage (RA), social influence (SI) and past experience with other smart devices (PE) are the strong predictors of the perceived usefulness of video functionality (PU-V) and they explained a total of $35.5 \%$ adjusted variance. SH, RA, SI and PE have positive impact on $\mathrm{PU}-\mathrm{V}$ and $\mathrm{SH}$ contributed to the largest total variance among them.

Table 4. Hierarchical regression analysis predicting PU-NV

\begin{tabular}{cccc}
\hline Predictor & $\begin{array}{c}\text { Incremental } \\
\text { (adjusted) } \mathrm{R}^{2}\end{array}$ & $\begin{array}{c}\text { Standardized } \\
\text { Coefficients }(\beta)^{1}\end{array}$ & Sig. $^{1}$ \\
\hline PQ & 0.000 & 0.047 & 0.491 \\
SH & 0.032 & -0.018 & 0.819 \\
PE & -0.004 & -0.010 & 0.888 \\
INV & $\mathbf{0 . 0 3}$ & $\mathbf{0 . 1 6 3}$ & $\mathbf{0 . 0 1 6} *$ \\
RA & $\mathbf{0 . 0 2 6}$ & $\mathbf{0 . 1 9 0}$ & $\mathbf{0 . 0 1 1} *$ \\
COM & -0.003 & 0.024 & 0.728 \\
SI & -0.002 & 0.055 & 0.444 \\
PEOU & $\mathbf{0 . 0 2 6}$ & $\mathbf{0 . 1 9 5}$ & $\mathbf{0 . 0 0 6 * *}$ \\
\hline Age & & 0.002 & 0.971 \\
Current Usage of & -0.002 & 0.080 & 0.205 \\
Smart TV & & & \\
\hline Total & $\mathbf{0 . 1 0 3}$ &
\end{tabular}

$* \mathrm{p}<0.05 ; * * \mathrm{p}<0.01 ; * * * \mathrm{p}<0.001$. 
The result in Table 4 shows that innovativeness (INV), relative advantage (RA) and perceived ease of use (PEOU) are the strong predictors of the perceived usefulness of non-video functionalities (PU-NV), but they explained a mere $10.3 \%$ of total variance. INV, PEOU and RA have significant positive impact on PU-NV. The nonvideo functionalities of smart TV is still in early stage and those with higher innovativeness will most likely try these functionalities and PEOU and RA is still a bottle neck.

\subsection{Predicting the Intention to Use}

Finally, we performed a hierarchical regression analysis, and tried to predict user's intention to adopt an embedded smart TV or smart set-top box by PEOU, PU-V and PU-NV. The results shows that PU-NV, PEOU, PU-V are all strong predictors for intention to use embedded smart TV, while PU-V is the only predictor for intention to use a smart set-top box. And by combining with the previous results, we built up the following smart TV user adoption model. The construct "compatibility" is not a strong predictor for none of PU-NV, PEOU and PU-V, and is eliminated from the model.

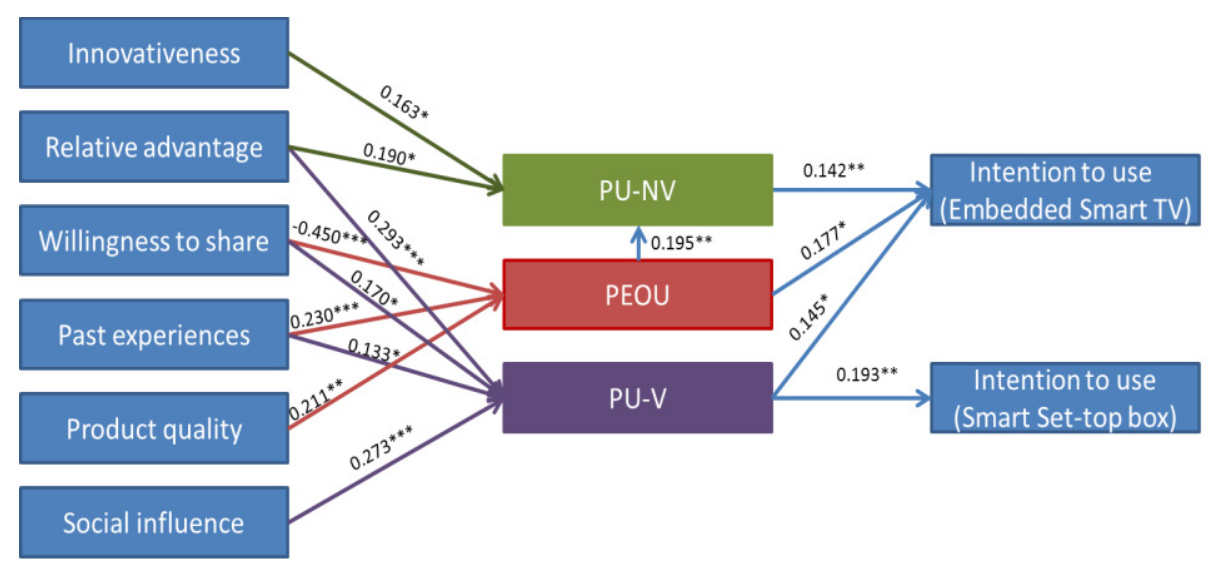

Fig. 2. Smart TV User Adoption Model

\section{Conclusion and Discussion}

In this research, we adapted the TAM model to explore the factors that impact Chinese users' intention to adopt two forms of smart TV: embedded smart TV and smart set-top box and how the adoptions of the two are different from each other. The construct of perceived usefulness was spitted into two in our model: perceived usefulness of video functionalities and perceived usefulness of non-video functionalities. Six factors were distinguished as the antecedents to perceived ease of use and the two perceived usefulness constructs. Based on the model we achieved, the following conclusions were drawn. 
Firstly, video functionality is more important than the other smart functionalities for smart TVs. The exploratory factor analysis divided the survey items for the construct "perceived usefulness" into two groups: one group related to video functionality, and the other group related to non-video functionalities and this led us to split perceived usefulness into two: perceived usefulness for video functionality and perceived usefulness for non-vide functionality. The mean of the former is significantly higher than the later. For Chinese TV manufactures, the first priory in developing a smart TV is to provide users with abundant online video contents to make their product useful to consumers. Other functionalities like TV games and TV shopping are not as important as online video in driving the consumers to make the purchase decision.

Secondly, even though smart set-top boxes are similar with embedded smart TV in terms of functionalities, the factors that impact the attention to adopt them are different. Embedded smart TVs are much higher in price than set-top box, so both the video functionality and the other smart functionalities are important. Besides, an embedded smart TV should be fully optimized to facilitate its usage. In comparison, users' expectation for smart set-top box is much lower. Only the usefulness of video functionality is a big concern. This finding instructs the TV manufactures to differentiate their embedded TV products and the smart box products. The latter should focus themselves on online video, while the former should be positioned to a relatively high end market and make themselves worth the price.

Thirdly, antecedent factors for perceived usefulness on video functionality and non-video functionalities are different. Those with satisfying experiences in using smart phones and smart tablets and a high willingness to share video contents together with families will most likely find the video functionality useful, while those who are highly innovative are most likely to find other smart functionalities useful. Social influence will affect users' opinion on usefulness of smart TV video, but not smart TV games, smart TV shopping etc. According to the innovation diffusion theory, the smart TV video and other functionalities are in different stage of diffusion. Smart TV video is gradually being accepted by the early adopter or even early majority in China, while other smart functionalities are still in the earliest stage of diffusion, thus only those highly innovative users are likely to try.

Finally, users with experience on smart phone and smart tablets are more likely to find smart TV useful and easy to use. The smart device users are potential target consumers of smart TVs. This finding is intuitive for both designing and marketing of smart TV products. For designer of smart TV, the product should be designed compatible with the habits of smart phone or smart tablets consumers. As well, internet channel, particularly the mobile internet is an ideal place to promote smart TV products, because the mobile internet user group coincides with that of the smart TVs.

There're also several limitations to the study. Firstly, we used the hierarchical regression analysis to build the model, but the total variances explained by the regression are quite low, this resulted in a lower reliability of the results and other factors that can better predict the user adoption might have been omitted. The current model still has large space for improvement. Secondly, the survey questionnaire is quite long and it spread online, the quality of the responses could not be fully guaranteed (with 
only $45.7 \%$ of effective rate) even though we've taken measures of precautions. A better design of the survey and more precautious selection of the subjects may lead to more convincing results.

\section{References}

1. Zillmann, D.: The coming of media entertainment. In: Zillmann, D., Vorderer, P. (eds.) Media Entertainment: The Psychology of Its Appeal, pp. 1-20. Erlbaum, Mahwah, NJ (2000)

2. Liu, J., Liu, Y., Rau, P.L.P., Li, H., Wang, X., Li, D.: How socio-economic structure influences rural users' acceptance of mobile entertainment. In: Proceedings of the SIGCHI Conference on Human Factors in Computing Systems, pp. 2203-2212. ACM (April 2010)

3. Cabrer, M.R., Redondo, R.P.D., Vilas, A.F., Pazos Arias, J.J., Duque, J.G.: Controlling the smart home from TV. IEEE Transactions on Consumer Electronics 52(2), 421-429 (2006)

4. Davis, F.D.: Perceived Usefulness, Perceived Ease of Use, and User Acceptance of Information Technology. MIS Quarterly 13(3), 319-339 (1989)

5. Everett, R.: Diffusion of innovations, vol. 5, pp. 11-27. The Free Press, New York (1995)

6. Bandura, A.: Self-Efficacy Mechanismin Human Agency. American Psychologist 37(2), 122-147 (1982)

7. Beach, L.R., Mitchell, T.R.: A Contingency Model for the Selection of Decision Strategies. Academy of Management Review 3(3), 439-449 (1978)

8. Tornatzky, L. G., Klein, K.J.: Innovation Characteristics and Innovation Adoption - Implementation: A Meta-Analysis of Findings. IEEE Transactions on Engineering Management 29(1), 28-45 (1982)

9. Venkatesh, V., Davis, F.D.: A theoretical extension of the technology acceptance model: four longitudinal field studies. Management Science 46(2), 186-204 (2000)

10. Park, C., Jun, J.K.: A cross-cultural comparison of Internet buying behavior: Effects of Internet usage, perceived risks, and innovativeness. International Marketing Review 20(5), 534-553 (2003)

11. Garcia, R., Calantone, R.: A critical look at technological innovation typology and innovativeness terminology: a literature review. Journal of Product Innovation Management 19(2), 110-132 (2002)

12. Lu, J., Yao, J.E., Yu, C.S.: Personal innovativeness, social influences and adoption of wireless Internet services via mobile technology. The Journal of Strategic Information Systems 14(3), 245-268 (2005)

13. De Ruyter, K., Wetzels, M., Kleijnen, M.: Customer adoption of e-service: an experimental study. International Journal of Service Industry Management 12(2), 184-207 (2001)

14. Shin, D.H., Hwang, Y., Choo, H.: Smart TV: are they really smart in interacting with people? Understanding the interactivity of Korean Smart TV. Behaviour \& Information Technology 32(2), 156-172 (2013)

15. Smith, B., Caputi, P., Rawstorne, P.: The development of a measure of subjective computer experience. Computers in Human Behavior 23(1), 127-145 (2007)

16. Hair, J.F., et al.: Multivariate data analysis: with readings. Macmillan (1995)

17. Stiggelbout, A.M., Molewijk, A.C., Otten, W., Timmermans, D.R.M., Van Bockel, J.H., Kievit, J.: Ideals of patient autonomy in clinical decision making: a study on the development of a scale to assess patients' and physicians' views. Journal of Medical Ethics 30(3), 268-274 (2004) 